\title{
Broadband Circular Polarizer Based on Multilayer Gradual Frequency Selective Surfaces
}

\author{
Wei Zhang, Jian-ying Li, and Ling Wang \\ School of Electronics and Information, Northwestern Polytechnical University, Xian 710072, China \\ Correspondence should be addressed to Wei Zhang; zhangwei19900410@163.com
}

Received 17 June 2016; Revised 8 August 2016; Accepted 21 August 2016

Academic Editor: Giuseppe Mazzarella

Copyright ( 2016 Wei Zhang et al. This is an open access article distributed under the Creative Commons Attribution License, which permits unrestricted use, distribution, and reproduction in any medium, provided the original work is properly cited.

\begin{abstract}
This paper presents a broadband circular polarizer based on multilayer gradual frequency selective surfaces. The unit cell includes two identical circles and a bar, whose dimensions decrease layer by layer with a reduction factor. This design can provide a good phase difference that is close to $90^{\circ}$. The bandwidth of the polarizer reaches $66.7 \%$, from 7.9 to $15.8 \mathrm{GHz}$, which can convert the incident linear polarization waves into circular polarization waves. A sample polarizer is fabricated and measured. The results show that the operation frequency ranges from 7.75 to $15.2 \mathrm{GHz}$, coinciding with the simulation. Meanwhile, the working frequency band covers the range of $8.2-14.5 \mathrm{GHz}$ when the incident angle increases to $25^{\circ}$.
\end{abstract}

\section{Introduction}

Circular polarizers, which converts linear polarization waves (LPWs) to circular polarization waves (CPWs), are of great importance in many applications where the diversity of polarization is desired [1]. For example, circular polarizers are applied in minimizing the effectiveness of Faraday rotation incurred by the ionosphere of the satellite systems [2]. Also, they can be utilized to mitigate echoes from rain or suppressing interference in communicational systems $[3,4]$. A variety of polarization converters have already been proven nowadays [5], for instance, alternate medium plate of various materials [6], four-arm structure [7], lattice structures utilizing rods or strips [8], and rectangular slot antenna [9]. However, most of these polarizers are limited by their operational bandwidth.

It is quite a good way to get CPW by employing frequency selective surfaces (FSS), which has been successfully practiced in former circular polarizers. Because of the low profile and the sample fabrication, FSS is more attractive among periodic structures.

As discussed in [10], a single layer polarizer is proposed for remote environmental monitoring, which can be conveniently implemented in the submillimeter wave range. The $3 \mathrm{~dB}$ axial ratios (AR) bandwidth of this polarizer is nearly $11.75 \%$. In [11], the bandwidth increases to $21 \%$ based on the same unit cell geometry. The polarizer in [12] is made up of Jerusalem-cross, which provides nearly $99 \%$ polarization purity at the operation frequency, yet the bandwidth is only $4.3 \%$. With a similar high purity, [13] offers a dual-band polarization convertor based on two concentric rings. The total bandwidth is up to $9.9 \%$ while the insertion loss increases to $5.1 \mathrm{~dB}$. In [14], the authors found that the insertion loss could be significantly reduced by adding one FSS layer. Table 1 shows the comparison of these circular polarizers.

In this paper, a broadband circular polarizer is presented based on multilayer gradual frequency selective surfaces (MG-FSS). The AR lower than $3 \mathrm{~dB}$ are over the frequency range from $7.9 \mathrm{GHz}$ to $15.8 \mathrm{GHz}$. Meanwhile, the polarizer can obtain the working frequency band over the range of 8.2$14.5 \mathrm{GHz}$ when the incident angle increases to $25^{\circ}$.

\section{Principle of Operation}

Polarization of a plane wave is the direction of its electric field vector, which may be in a fixed orientation or might alter over time. CPW is featured by electric field in which the two orthogonal parts have the same amplitude and a phase difference of $90^{\circ}$. A LPW passes through a polarizer, which offers such feature between two orthogonal parts. In this way, a CPW is obtained. 
TABLE 1: Comparison of circular polarizers.

\begin{tabular}{lccc}
\hline Ref. & $\begin{array}{c}\text { Size of unit cell } \\
\left(\times \lambda^{3} \mathrm{~mm}^{3}\right)\end{array}$ & $\begin{array}{c}\text { Frequency } \\
(\mathrm{GHz})\end{array}$ & $\begin{array}{c}\mathrm{AR}<3 \mathrm{~dB} \\
\text { Bandwidth }(\%)\end{array}$ \\
\hline$[10]$ & $0.51 \times 0.59 \times 0.01$ & 325 & 11.75 \\
{$[11]$} & $0.51 \times 0.59 \times 0.01$ & 325 & 21 \\
{$[12]$} & $0.33 \times 0.33 \times 0.09$ & 17.8 & 4.3 \\
{$[13]$} & $0.56 \times 0.56 \times 0.08$ & 27.21 & 5.6 \\
{$[14]$} & $0.60 \times 0.52 \times 0.03$ & 10 & 4.3 \\
This paper & $0.3 \times 0.6 \times 0.24$ & 12 & 6.8 \\
\hline
\end{tabular}

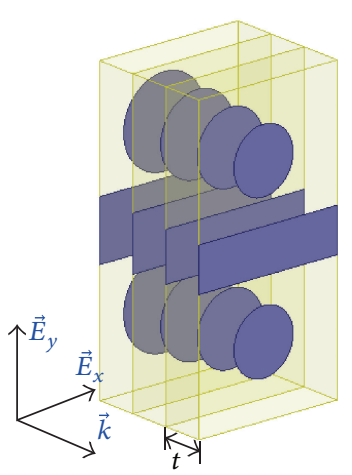

(a)

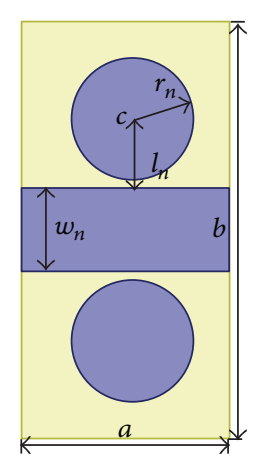

(b)
FIGURE 1: (a) The unit cell of the MG-FSS. (b) Geometry of each layer.

The proposed circular polarizer is shown in Figure 1(a). Each layer consists of two identical circles and a bar in the middle of the unit. We superimpose a four-layer architecture, which can achieve not only the desired phase difference but also a good transmission coefficient. It should be noted that the size of the FSS strip in each layer varies gradually. Figure 1(b) presents the front view of the metal strip, where $n$ denotes the $n$th layer. The first is the biggest one, and the other three are diminishing according to the reduction factor $\eta$.

Consider an incident LPW traveling along the $-z$ direction, whose electric field $\vec{E}^{i}$ is slant at $\psi=45^{\circ}$ to the $x$-axis. The incident LPW can be decomposed into two even parts in horizontal and vertical directions accordingly:

$$
\vec{E}^{i}=\vec{E}_{x}^{i}+\vec{E}_{y}^{i}=E_{0}(\hat{x}+\hat{y}) e^{j k z},
$$

where the unit vectors $\hat{x}$ and $\hat{y}$ are parallel and perpendicular to the metal bar, respectively. Generally, the incident LPW can be decomposed into $\vec{E}_{x}^{i}$ and $\vec{E}_{y}^{i}$, which are in the same phase and magnitude. Ideally, the two parts will be completely transmitted without reflection and insertion loss. One step further, the FSS provides an inductive behavior for $\vec{E}_{x}^{i}$ mainly because of the metal bar in $x$-direction, while the FSS becomes capacitive for $\vec{E}_{y}^{i}$ due to the nonmetal in $y$-direction. The transmitted wave can also be presented as a sum of

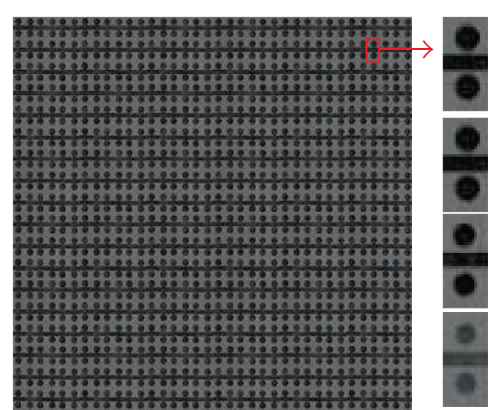

FIGURE 2: The fabricated polarizer and the four layers of the unit. The size is diminished from the top down.

two orthogonal linearly polarized components with equal magnitudes:

$$
\vec{E}^{t}=\vec{E}_{x}^{t}+\vec{E}_{y}^{t}=E_{0}\left(T_{x} \hat{x}+T_{y} \hat{y}\right) e^{j k z},
$$

where $T_{x}=\left|T_{x}\right| e^{j \varphi_{x}}$ and $T_{y}=\left|T_{y}\right| e^{j \varphi_{y}}$ are the transmission coefficients of $\vec{E}_{x}^{i}$ and $\vec{E}_{y}^{i}$, respectively. The metal bar in $x$ direction and the nonmetal in $y$-direction provide different transmission characteristics for the two orthogonal electric field vectors. The phase of the $x$-direction part is delayed by $\varphi_{x}$, while that of the $y$-direction part is advanced by $\varphi_{y}$. Thus, a phase difference $\Delta \varphi=\left|\varphi_{x}-\varphi_{y}\right|$ appears between the two components at the output of the polarizer. If the parameters of the polarizer are adjusted to satisfy $\left|T_{x}\right|=\left|T_{y}\right|$ and $\Delta \varphi=90^{\circ}$, then CPW can be produced.

\section{Optimization Design and Manufacturing}

The proposed polarizer operates in X-band. In the following experiments, a four-layer polarizer, with each layer consisting of $18 \times 36$ elements, is fabricated and tested. The metallic layers are modeled as a $0.05 \mathrm{~mm}$ copper film with an electric conductivity $\sigma=5.8 \times 10^{7} \mathrm{~S} / \mathrm{m}$. The substrate is selected as Arlon Diclad 880 with a relative permittivity of 2.2 and a dielectric loss tangent of 0.009 , whose thickness is $t=2 \mathrm{~mm}$. Figure 2 shows the sample polarizer. As shown in Figure 1, the dimensions of the biggest layer are $a=7.5 \mathrm{~mm}, b=15 \mathrm{~mm}$, $l_{1}=2.5 \mathrm{~mm}, w_{1}=3 \mathrm{~mm}$, and $r_{1}=2.4 \mathrm{~mm}$. The reduction factor $\eta=0.9$, which means that the size of the metal strip in one layer reduces to $90 \%$ compared to that of the previous layer, while the location is a constant.

A vector network analyzer (VNA) is applied to measure the polarizer, which is in connection with the two standard LP horn antennas which radiate electromagnetic waves in a broadband of 7-16 GHz. The horn antennas are aligned for maximum reception and the transmitting one is set to provide a LPW whose electric field is oriented $45^{\circ}$ to the $x$-axis. The prototype has been put in the middle between two antennas. Besides, all the helpful information of transmission for various polarizations can be obtained through transforming the directions of the two LP horn antennas. 


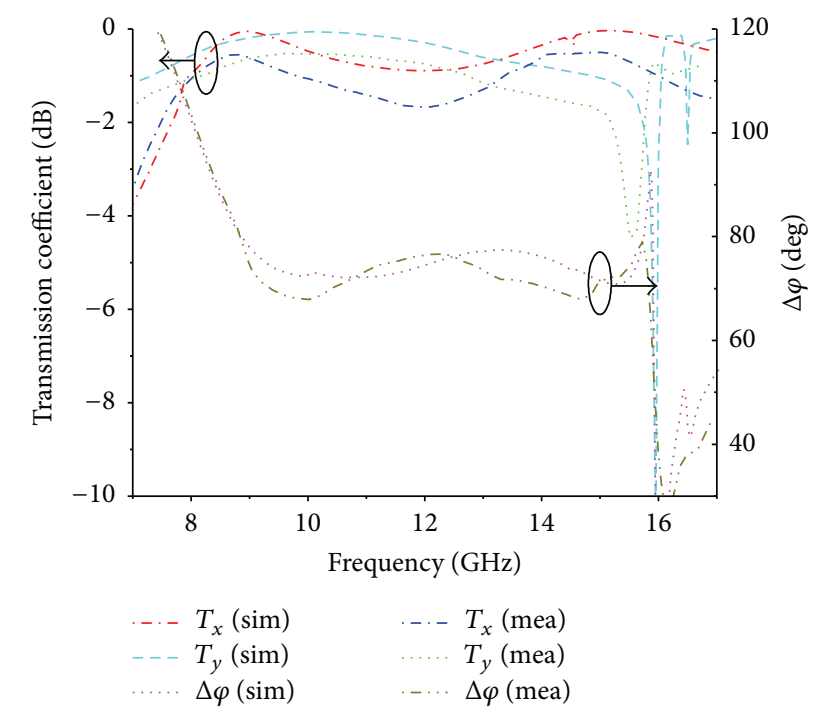

FIgURE 3: The simulated and measured magnitudes of the transmission coefficients $T_{x}$ and $T_{y}$, and the phase difference $\Delta \varphi$.

In order to better illustrate the polarized conversion of the MG-FSS discussed in the paper, the axial ratio is calculated as

$$
\begin{aligned}
\mathrm{AR} & =\left[\frac{(1 / a) \cos ^{2} \tau+\sin 2 \tau \cos \Delta \varphi+a \sin ^{2} \tau}{(1 / a) \sin ^{2} \tau-\sin 2 \tau \cos \Delta \varphi+a \cos ^{2} \tau}\right] \\
a & =\frac{\left|\vec{E}_{x}^{t}\right|}{\left|\vec{E}_{y}^{t}\right|} \\
\tan 2 \tau & =\frac{2 a}{1-a} \cos \Delta \varphi,
\end{aligned}
$$

where $\left|\vec{E}_{x}^{t}\right|$ and $\left|\vec{E}_{y}^{t}\right|$ refer to the maximum magnitudes of the horizontal and vertical transmission parts in the electric field. The inclination angle $\tau$ of the ellipse can be generated by (5).

The magnitudes of the transmission coefficients $T_{x}$ and $T_{y}$ are shown in Figure 3, as well as the phase difference $\Delta \varphi$. Due to the measured conclusion for finite units instead of an infinite periodic structure in simulation, there is a little bit of deflection between the simulated and measured results. Figure 4 shows the simulated and measured AR of the transmitted wave. A rational agreement between the simulated and measured results is detected. The operation bandwidth of the proposed structure will reach $66.7 \%$, ranging from $7.9 \mathrm{GHz}$ to $15.8 \mathrm{GHz}$. The actual measurement results indicate that the fabricated device can work as a circular polarizer over the frequency range from $7.75 \mathrm{GHz}$ to $15.2 \mathrm{GHz}$. The differences between numerical simulations and experimental results can be explained by the fact that the measured results are for the finite elements while the simulation results are for infinite periodic units. Moreover, machining tolerance, border effects, and slight ellipticity of the incident LPW may also deteriorate the measured results.

For better illustration, polarization ellipses are plotted on the basis of the measure results. Eight uniformly spaced frequency points along the working band are plotted to

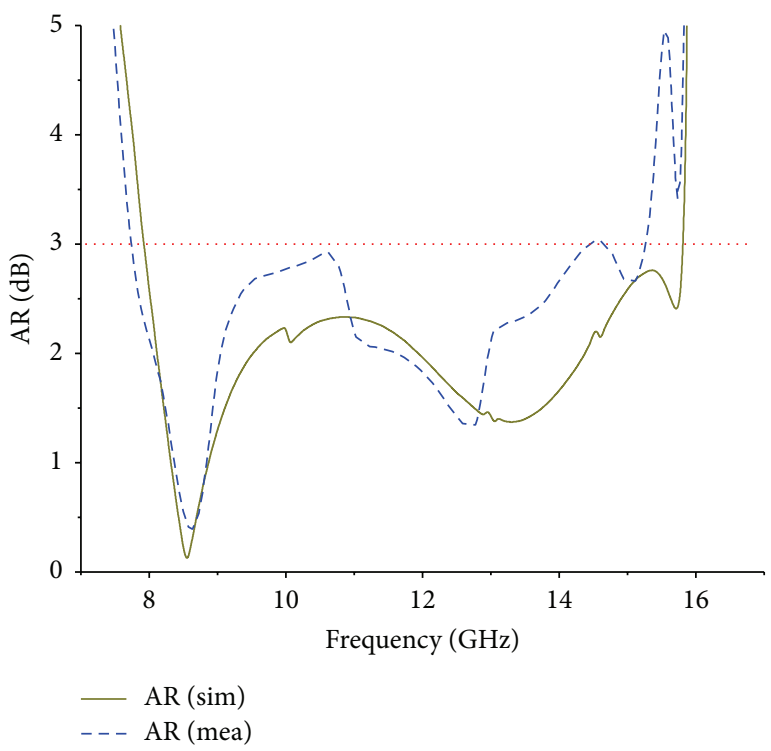

FIgURE 4: The simulated and measured AR.

observe the polarization. Figure 5 shows the ellipses of the CPW obtained at $8-15 \mathrm{GHz}$ with a step of $1 \mathrm{GHz}$.

\section{Discussion}

4.1. Oblique Incidence. The transmission features of any periodic structure would vary according to the change of incidence angle $\theta_{\text {inc }}$ Different angles of oblique incidence are assessed. It can be seen from Figure 6 that as $\theta_{\text {inc }}$ increases, the bandwidth varies slightly while the AR changes remarkably. It is notable that such deterioration proves to be more pronounced for higher frequencies. In addition, the AR close to the center frequency is stable. The experimental results show that the proposed circular polarizer can make the AR under $3 \mathrm{~dB}$ over the range of $8.2-14.5 \mathrm{GHz}$, when the incident angle increases to $25^{\circ}$.

4.2. Reduction Factor. One of the most important parameters in the designed polarizer is the reduction factor $\eta$. Figure 7 displays the simulated variation as $\eta$ changes from 0.7 to 1 . It is clear to see that the reduction factor has a large impact on the performance. Circular polarization characteristics gradually get better with the increase of $\eta$. However, the AR becomes unsatisfactory when the reduction factor reaches 1. It means that the structure could no longer be used as a circular polarizer if the four layers are the same. Extensive experiments show that the main reason is the phase difference of the two orthogonal components of the transmitted waves. In the second section, we discuss the relationship between the circular polarization and the phase difference. For this structure, the electromagnetic waves will produce certain phase difference $\Delta \varphi_{n}$ through each layer and different size generates different phase difference. When the waves pass through the entire polarizer, the difference between the two orthogonal components of the transmitted waves $\Delta \varphi$ is the superposition of all $\Delta \varphi_{n}$. It should be noted that $\Delta \varphi_{n}$ are not 


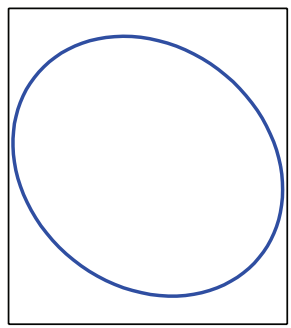

$8 \mathrm{GHz}$

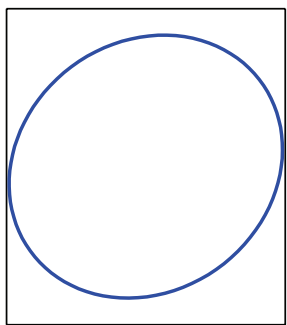

$12 \mathrm{GHz}$

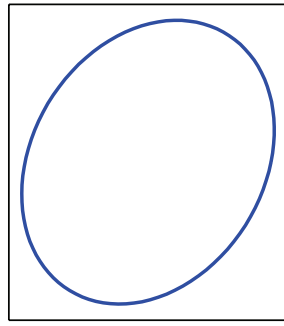

$9 \mathrm{GHz}$

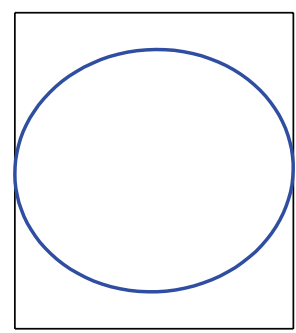

$13 \mathrm{GHz}$

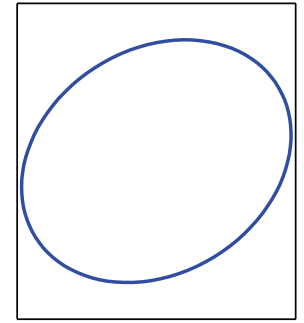

$10 \mathrm{GHz}$

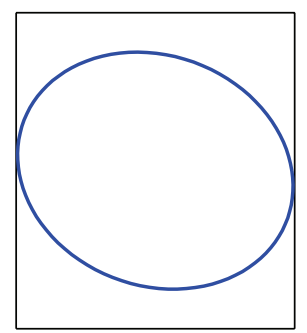

$14 \mathrm{GHz}$

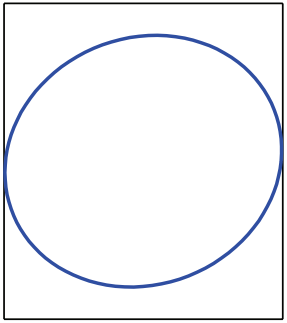

$11 \mathrm{GHz}$

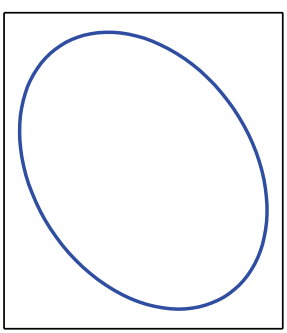

$15 \mathrm{GHz}$

FIGURE 5: Polarization ellipses of the proposed polarizer based on the measure results.

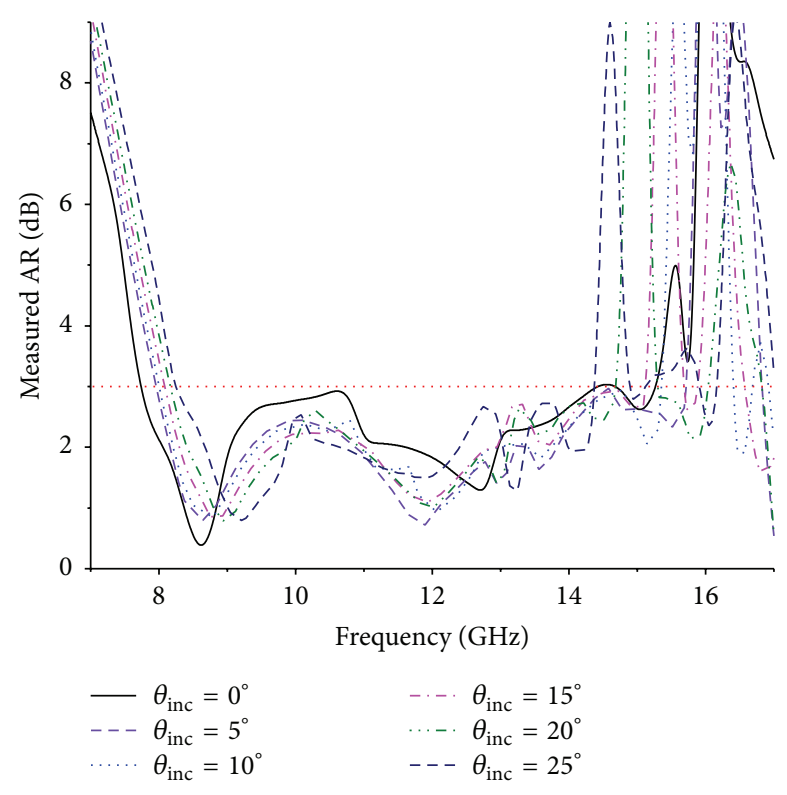

FIGURE 6: The simulated and measured AR.

identical to each other because of the coupling of layers and their different dimensions.

According to the simulation results, the waveforms of the two orthogonal waves through each layer are presented. Figure 8 shows the waveforms at $12 \mathrm{GHz}$ when $\eta=0.9$ and $\eta=1$, respectively. $E_{x}^{i}$ is the incident wave; $E_{x}^{n}$ and $E_{y}^{n}(n=$ $1,2,3)$ denote the electric fields through the $n$th layer in the $x$ direction and $y$ direction, respectively. $E_{x}^{t}$ represents the electric field through the fourth layer, which is also the transmitted wave. Figure 8 displays that periodic units of each layer produce discrepant effect on the two parts. When the waves pass through the fourth layer, $\Delta \varphi$ approximates $90^{\circ}$ if

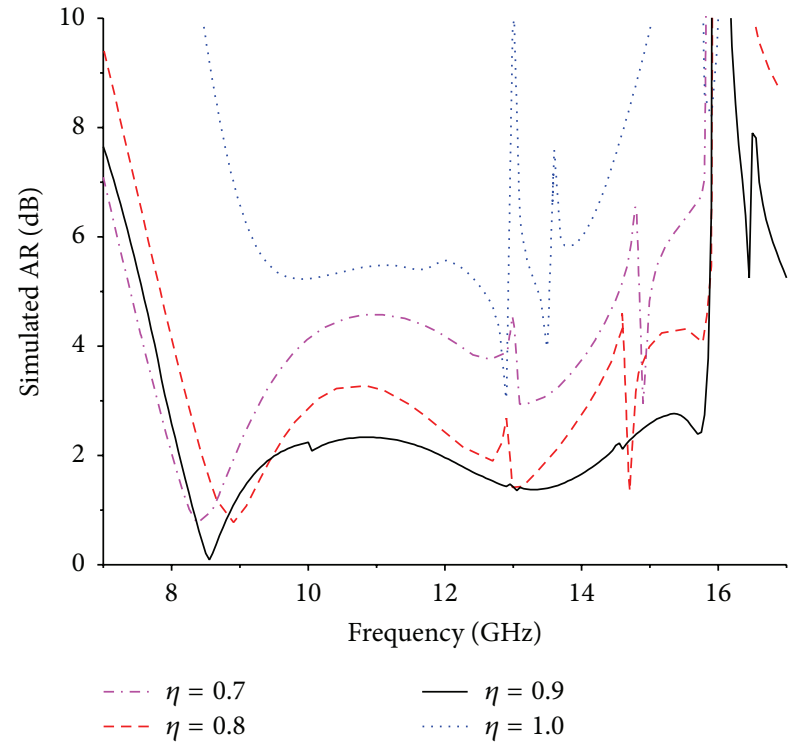

FIGURE 7: The simulated variation as the reduction factor.

$\eta=0.9$. In this way, a LPW can be successfully converted into a CPW. If all the layers are the same, $\Delta \varphi$ is only about $55^{\circ}$. Therefore, the structure cannot be used as a circular polarizer any more.

It is worth noting that, in the process of the experiment, we also found that the proposed polarizer does not exist positively or negatively. That is, whether the propagation of electromagnetic waves is $+z$ or $-z$, the results are the same.

\section{Conclusion}

A broadband circular polarizer based on multilayer gradual frequency selective surfaces has been presented in this paper. 

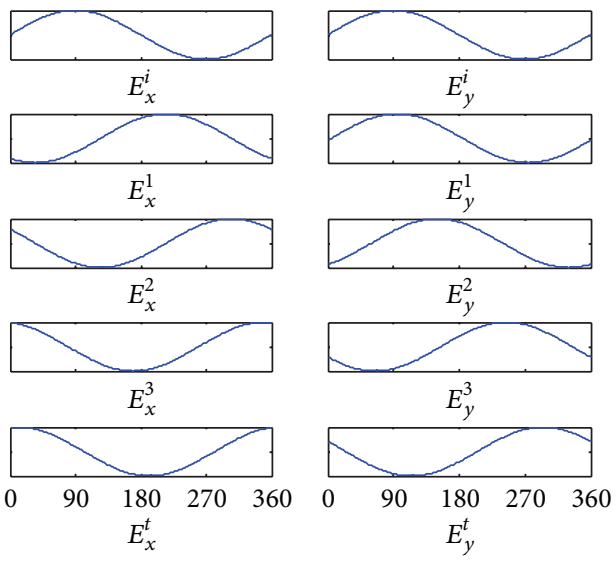

(a)
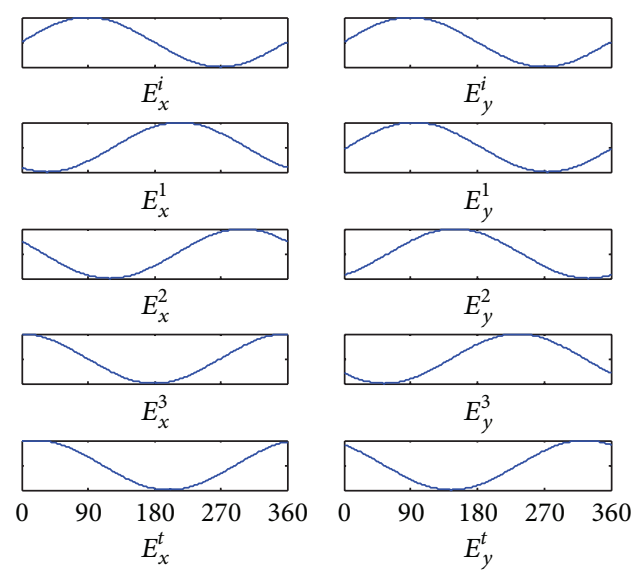

(b)

FIGURE 8: The waveforms at $12 \mathrm{GHz}$ : (a) reduction factor is 0.9 ; (b) reduction factor is 1.

The investigated results show that the $3 \mathrm{~dB}$ AR bandwidth for the proposed polarizer reaches $66.7 \%$ at normal incidence from 7.9 to $15.8 \mathrm{GHz}$. Also, a sample polarizer is fabricated and tested. The measured results show that the sample can operate from 7.75 to $15.2 \mathrm{GHz}$, which is in a reasonable agreement with simulation results. Meanwhile, the polarizer can obtain a working frequency band over the range of $8.2-14.5 \mathrm{GHz}$ when the incident angle is as high as $25^{\circ}$.

\section{Competing Interests}

The authors declare that they have no competing interests.

\section{Acknowledgments}

This work was supported in part by the National Natural Science Foundation of China (nos. 61271416 and 61301093), the Fundamental Research Funds for the Central Universities (no. 3102014KYJD027), and NPU Foundation for Fundamental Research (no. JCY20130132). The authors would like to thank Wei Kun and Xu Rui for their kind assistance with the test.

\section{References}

[1] K. S. Min, J. Hirokawa, K. Sakurai, and M. Ando, "Single-layer dipole array for linear-to-circular polarisation conversion of slotted waveguide array," Microwaves, Antennas and Propagation, vol. 143, no. 13, pp. 211-216, 1996.

[2] G. Maral and M. Bousquet, Satellite Communications SystemsSystems, Techniques and Technology, John Wiley \& Sons, Sussex, UK, 5th edition, 2009.

[3] G. Liu, L. Xu, and Y. Wang, "Modified dual-band stacked circularly polarized microstrip antenna," International Journal of Antennas and Propagation, vol. 2013, Article ID 382958, 5 pages, 2013.

[4] C. Liu, A. Yan, C. Yu, and T. Xu, "Improvement on a $2 \times 2$ elements high-gain circularly polarized antenna array," International Journal of Antennas and Propagation, vol. 2015, Article ID 252717, 8 pages, 2015.
[5] C. Dietlein, A. Luukanen, Z. Popović, and E. Grossman, "A Wband polarization converter and isolator," IEEE Transactions on Antennas and Propagation, vol. 55, no. 6, pp. 1804-1809, 2007.

[6] J. Bornemann, "Computer-aided design of multilayered dielectric frequency-selective surfaces for circularly polarized millimeter-wave applications," IEEE Transactions on Antennas and Propagation, vol. 41, no. 11, pp. 1588-1591, 1993.

[7] S. X. Ta and I. Park, "Dual-band operation of a circularly polarized four-arm curl antenna with asymmetric arm length," International Journal of Antennas and Propagation, vol. 2016, Article ID 3531089, 10 pages, 2016.

[8] D. S. Lerner, "A wave polarization converter for circular polarization," IEEE Transactions on Antennas and Propagation, vol. 13, no. 1, pp. 3-7, 1965.

[9] B. Li, Y. Ding, and Y.-Z. Yin, "A novel dual-band circularly polarized rectangular slot antenna," International Journal of Antennas and Propagation, vol. 2016, Article ID 9071610, 8 pages, 2016.

[10] M. Euler, V. Fusco, R. Cahill, and R. Dickie, “ $325 \mathrm{GHz}$ single layer sub-millimeter wave FSS based split slot ring linear to circular polarization convertor," IEEE Transactions on Antennas and Propagation, vol. 58, no. 7, pp. 2457-2459, 2010.

[11] M. Euler, V. Fusco, R. Dickie, R. Cahill, and J. Verheggen, "Sub$\mathrm{mm}$ wet etched linear to circular polarization FSS based polarization converters," IEEE Transactions on Antennas and Propagation, vol. 59, no. 8, pp. 3103-3106, 2011.

[12] I. Sohail, Y. Ranga, K. P. Esselle, and S. G. Hay, "A linear to circular polarization converter based on Jerusalem-Cross frequency selective surface," in Proceedings of the 7th European Conference on Antennas and Propagation (EuCAP '13), pp. 21412143, Gothenburg, Sweden, April 2013.

[13] Y. Ranga, L. Matekovits, S. G. Hay, and T. S. Bird, "An anisotropic impedance surface for dual-band linear-to-circular transmission polarization convertor," in Proceedings of the International Workshop on Antenna Technology (iWAT '13), pp. 47-50, Karlsruhe, Germany, March 2013.

[14] M. Euler, V. Fusco, R. Cahill, and R. Dickie, "Comparison of frequency-selective screen-based linear to circular split-ring polarisation convertors," IET Microwaves, Antennas and Propagation, vol. 4, no. 11, pp. 1764-1772, 2010. 


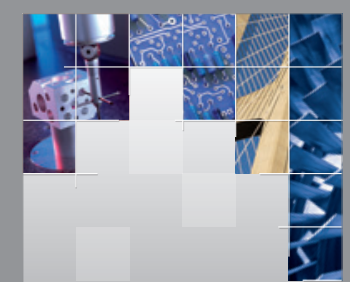

\section{Enfincering}
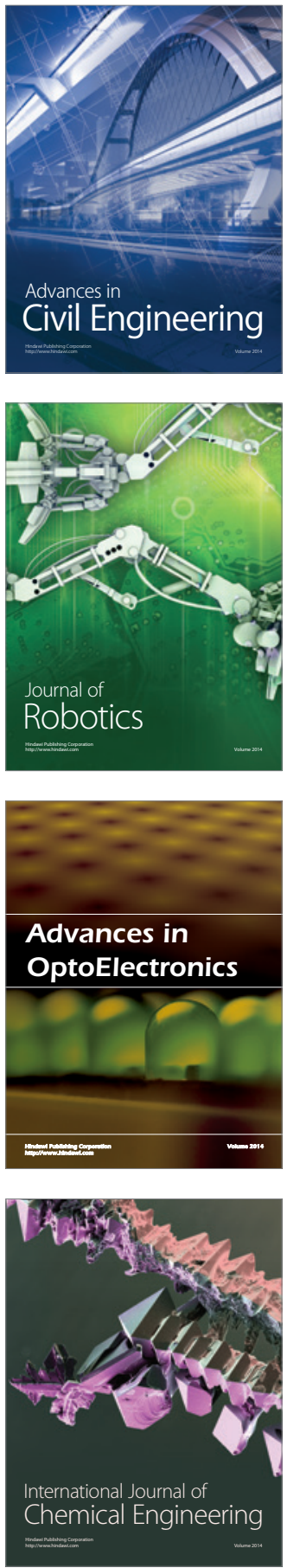

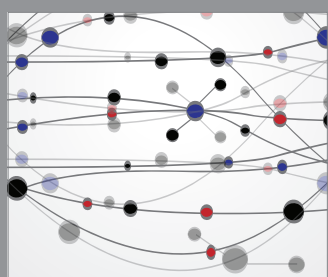

The Scientific World Journal

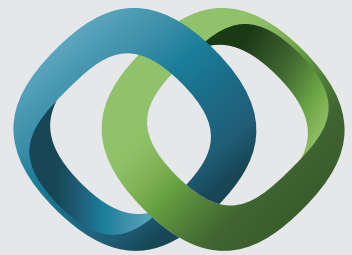

\section{Hindawi}

Submit your manuscripts at

http://www.hindawi.com
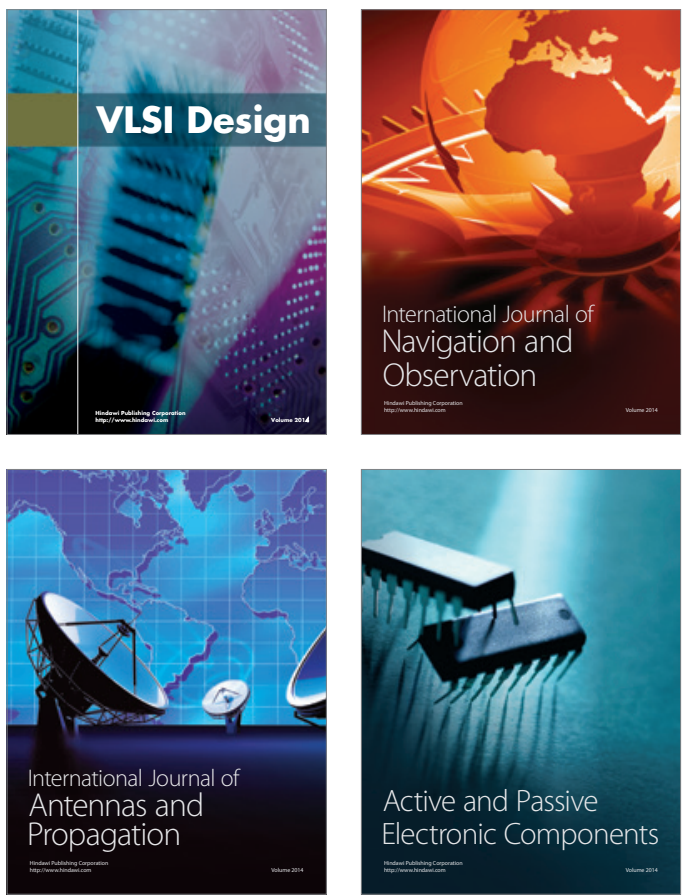
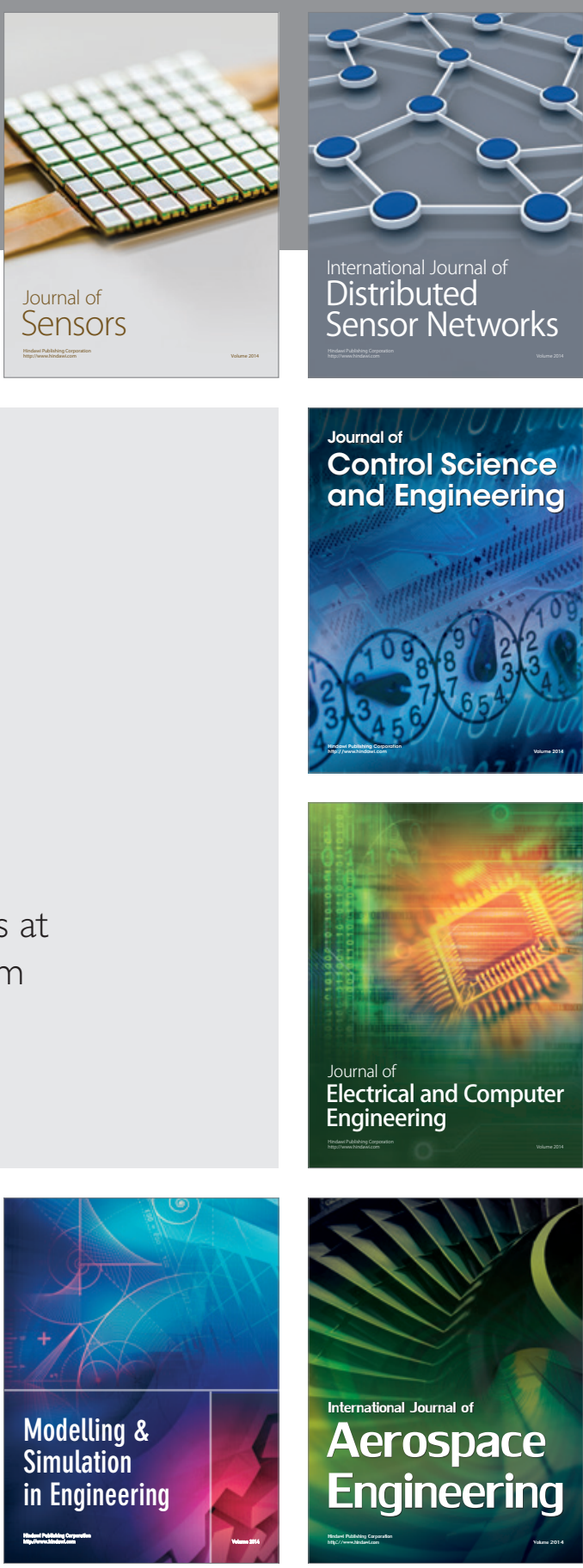

International Journal of

Distributed

Sensor Networks

Journal of

Control Science

and Engineering
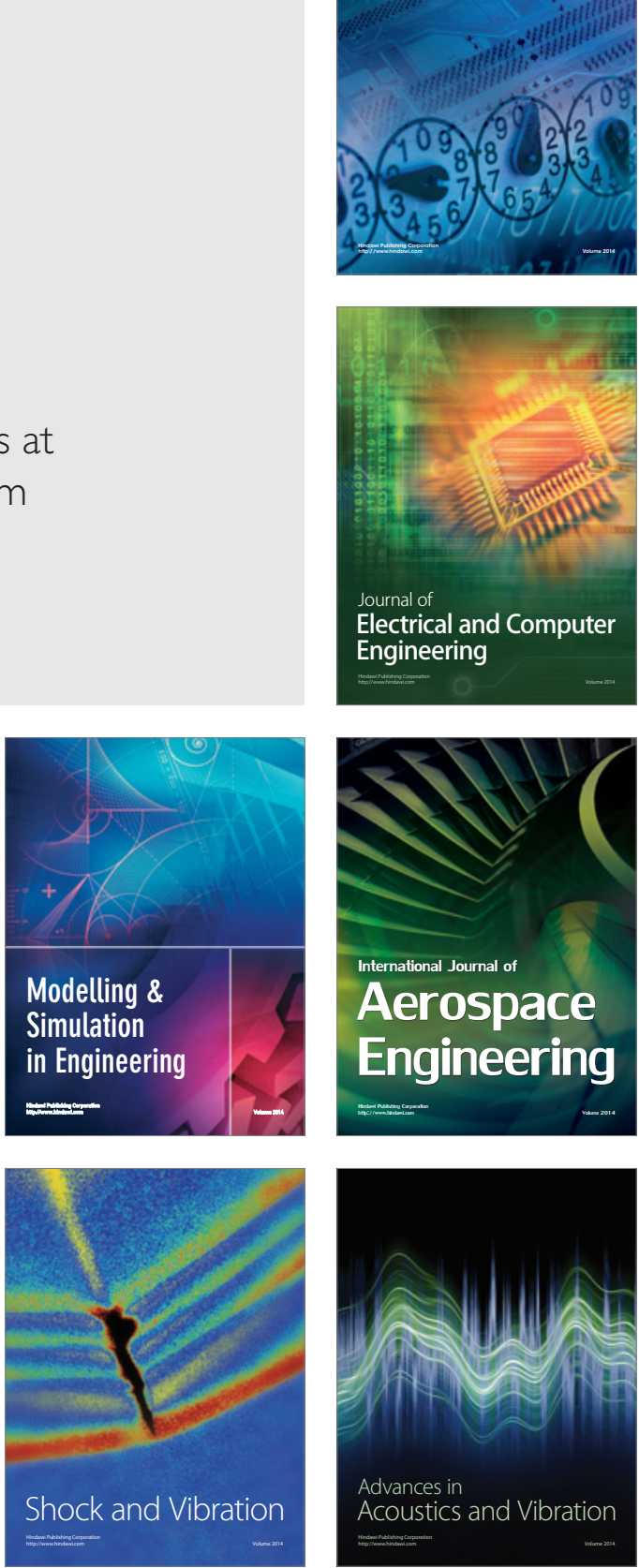